\title{
Metabolome and microbiome of chronic periapical periodontitis in permanent anterior teeth: a pilot study
}

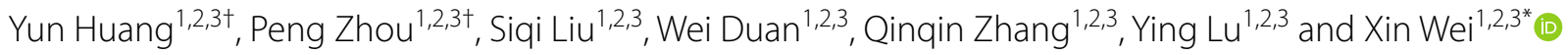

\begin{abstract}
Background: Periapical periodontitis is a common oral inflammatory disease that affects periapical tissues and is caused by bacteria in the root canal system. The relationship among the local metabolome, the inflammatory grade, and the type and abundance of microorganisms associated with periapical periodontitis is discussed in this study.

Methods: The inflammatory grades of periapical samples from 47 patients with chronic periapical periodontitis in permanent anterior teeth were determined based on the immune cell densities in tissues subjected to haematoxylin and eosin staining. The metabolome was evaluated using ultrahigh-performance liquid chromatography-quadrupole time-of-flight mass spectrometry, followed by principal component analysis and orthogonal partial least squares discriminant analysis. The microbiome was accessed using $16 \mathrm{~S}$ rRNA high-throughput sequencing. The differences in the metabolomes and microbiomes of the periapical periodontitis samples were assessed using Spearman's correlation analysis.
\end{abstract}

Result: $N$-acetyl-D-glucosamine, L-tryptophan, L-phenylalanine, and 15 other metabolites were identified by the comparison between samples with severe inflammation and mild or moderate inflammation. Four amino acid metabolism pathways and one sugar metabolism pathway were associated with the inflammatory grade of periapical periodontitis. The abundance of Actinomycetes was negatively correlated with the abundance of glucosamine (GlcN), while the abundance of Tannerella was positively correlated with the abundance of L-methionine.

Conclusions: The local metabolome of periapical periodontitis is correlated with the inflammatory grade. The abundance of the local metabolites GlcN and L-methionine is correlated with the abundance of the major microorganisms Actinomycetes and Tannerella, respectively.

Keywords: Periapical periodontitis, Inflammatory grades, Metabolome, Microbiome

\section{Background}

Periapical periodontitis is a common oral inflammatory disease that affects periapical tissues and is mainly

\footnotetext{
*Correspondence: weixinart@163.com

†Yun Huang \& Peng Zhou are contributed equally to this work and should be considered co-first authors

${ }^{2}$ Department of Operative Dentistry and Endodontics, Affiliated

Stomatological Hospital of Nanjing Medical University, Nanjing, Jiangsu,

China

Full list of author information is available at the end of the article
}

caused by bacteria inside the root canal system; periapical periodontitis leads to periodontal ligament destruction, alveolar bone resorption, and granulation tissue formation [1]. When the alveolar bone is heavily damaged and absorbed during periapical periodontitis and inflammation cannot be cured by root canal therapy alone, surgical resection is needed [2]. For cases that are difficult to cure with periapical surgery, it is necessary to remove the affected teeth and eliminate the source of infection in order to return the condition of 
the patient to normal. In addition, the infectious bacteria and virulence factors associated with some clinically severe periapical diseases can lead to subacute infected endocarditis or even septicemia in immunodeficient or immunocompromised patients and transplant patients, and these consequences can be life-threatening [3]. Due to the severe prognosis of periapical periodontitis, the mechanisms underlying the development of this disease need to be further studied.

Previous studies have indicated that there are many kinds of microorganisms in periapical periodontitis [4], and immune cells accumulate around the periapical tissue and secrete proinflammatory cytokines after tissue infection or injury [5]. Lymphocytes secrete proinflammatory cytokines, such as tumour necrosis factor (TNF), interleukin-1 (IL-1) and interleukin-6 (IL-6), and these cytokines can directly or indirectly stimulate the activity of osteoclasts and result in alveolar bone absorption, suggesting a possible role of immune cells in alveolar bone absorption [4].

Metabolism is a general term for chemical reactions that sustain life, and it mainly includes glucose metabolism, lipid metabolism, and amino acid metabolism. Microorganisms and local immune alterations affect local metabolism, and local metabolic changes might in turn affect inflammatory grades. For example, tryptophan is broken down into kynurenine under the action of indoleamine 2,3-dioxidase (IDO) in chronic nephritis, and the activity of IDO is positively correlated with the serum level of kynurenine and the severity of chronic nephritis [6]. Interactions between metabolism and inflammatory factors have been described, and inflammation could cause corresponding metabolic changes [7]. Metabolomics is a new approach that allows the simultaneous qualitative and quantitative analysis of all low-molecular-weight metabolites $(<1 \mathrm{kDa})$ in biological samples that are produced during an inflammatory or specific physiological period $[8,9]$. Previous studies revealed unique metabolic changes in patients with primary sclerosing cholangitis through the metabolomics analysis of portal venous and bile, providing a potential target for the development of new therapies [9]. The possible role of local metabolic changes caused by host immune/inflammatory alterations in response to periapical periodontitis needs to be discussed.

Periapical periodontitis is an oral inflammatory disease that affects periapical tissues and is caused by bacteria; however, little information is available in the scientific literature about the correlation between the local microbiome and the levels of inflammation associated with periapical periodontitis. Here, we investigated the relationship among the local metabolic differences, the inflammatory grade, and the type and abundance of microorganisms associated with periapical periodontitis. This study will provide potential targets for new strategies to prevent the development of and resolve periapical periodontitis.

\section{Materials and methods \\ Patient selection}

In this study, 47 patients (age 18 to 45 years) with chronic periapical periodontitis in anterior teeth after root canal therapy were enrolled from March 2018 to January 2020 from the Department of Endodontics of Jiangsu Stomatological Hospital (Nanjing, China). The diagnosis of chronic periapical lesions was confirmed by two endodontic specialists, and all the patients provided written informed consent. Patients who presented with systemic diseases, such as hypertension and diabetes, or recently had a history of antibiotic use were excluded from the study.

\section{Sample procedure}

Periapical tissues were collected during the process of periapical microsurgery, immediately washed and stored in sterile phosphate-buffered saline. Each periapical tissue sample was divided into 3 parts. One part of the tissue was incubated in $10 \%$ formalin solution for histopathological analysis. One part of the tissue was frozen in liquid nitrogen within $45 \mathrm{~min}$ and stored at $-80{ }^{\circ} \mathrm{C}$ for metabolome analysis. One part of the tissue was frozen in liquid nitrogen within $45 \mathrm{~min}$ and stored at $-80{ }^{\circ} \mathrm{C}$ for microbiome analysis.

\section{Histopathological analysis Haematoxylin and eosin (H\&E) staining}

The periapical specimens were fixed in $10 \%$ formalin buffer for $72 \mathrm{~h}$. Then, they were washed in running water for $2 \mathrm{~h}$, dehydrated in ascending dilutions of ethanol (70\% ethanol, 95\% ethanol for $30 \mathrm{~min}$, anhydrous ethanol I and anhydrous ethanol II for $40 \mathrm{~min}$ ), diazotized in xylene (xylene I, xylene II for $20 \mathrm{~min}$, and xylene III for $40 \mathrm{~min}$ ), and finally embedded in paraffin. Paraffin Sect. $(5 \mu \mathrm{m}$ thick) were heat immobilized, deparaffinized with xylene, and rehydrated using a graded series of ethanol. H\&E staining was performed, and then, the periapical tissues were observed with an optical microscope at 200 times magnification (Leica, Germany).

\section{Inflammatory grade}

The degree of inflammation in periapical periodontitis was divided into 3 levels based on the densities of immune cells in the tissues as observed under an optical microscope: grade I, inflammatory cells accounted for less than $1 / 3$ of each field; grade II, inflammatory cells accounted for $1 / 3$ to $2 / 3$ of each field; and grade III, 
inflammatory cells accounted for more than $2 / 3$ of each field [10].

\section{Metabolome analysis Metabolite extraction}

A 30-mg piece of periapical specimen collected from microapical surgical resection was placed in a $1.5-\mathrm{mL}$ Eppendorf tube with $600 \mu \mathrm{L}$ methanol $(2: 2: 1 \mathrm{v} / \mathrm{v} / \mathrm{v} ; 2 \mu \mathrm{g} /$ $\mathrm{mL}$ 2-chloro-L-phenylalanine as the internal standard) and then vortexed for $30 \mathrm{~s}$. Porcelain beads were added to the mixture, and a mixed grinding apparatus (JXFSTPRP-24, Shanghai, China) was used to homogenize the sample ( $45 \mathrm{~Hz}, 4 \mathrm{~min})$. Subsequently, ultrasonic treatment in an ice water bath was performed for $5 \mathrm{~min}$ and repeated 3 times. The sample was incubated at $-20{ }^{\circ} \mathrm{C}$ for $1 \mathrm{~h}$. Then, the mixture was centrifuged at $12,000 \mathrm{rpm}$ at 4 ${ }^{\circ} \mathrm{C}$ for $15 \mathrm{~min}$. Then, $200 \mu \mathrm{L}$ supernatant was transferred to a new Eppendorf tube for vacuum drying, mixed with $200 \mu \mathrm{L}$ extract (acetonitrile water volume ratio: 1:1), vortexed for $30 \mathrm{~s}$, and subjected to ultrasonic treatment in an ice water bath for $10 \mathrm{~min}$. Next, each sample was centrifuged at $12,000 \mathrm{rpm}$ at $4{ }^{\circ} \mathrm{C}$ for $15 \mathrm{~min}$. After centrifugation, an aliquot of $75 \mu \mathrm{L}$ supernatant was transferred to a sample vial $(2 \mathrm{~mL})$ for UHPLC/QTOF-MS (LC-MS) analysis. The quality control $(\mathrm{QC})$ sample was prepared by mixing equal amounts of $10 \mu \mathrm{L}$ from each sample, which together represented a QC standard for all the samples of periapical periodontitis. Then, $75 \mu \mathrm{L}$ of each mixture was used for testing. To assess the repeatability of the analysis, the QC sample was injected once after every five samples tested during acquisition [11].

\section{Metabolomic profiling technology}

LC-MS analysis of periapical specimens was carried out on an Agilent 1290 Infinity UHPLC system (Agilent, California, America) in positive mode and negative mode [12]. The chromatographic column was a UPLCBEHAmide column $(1.7 \mathrm{~mm}, 2.1 * 100 \mathrm{~mm}$, Waters, Milford, MA, USA). The optimal mobile phase was composed of "A" (25 mM ammonium acetate and $25 \mathrm{mM}$ ammonium hydroxide in water ( $\mathrm{pH}$ 9.75)) and "B" (acetonitrile). The gradient elution of the samples was performed as follows: $0-0.5 \mathrm{~min}, 5 \% \mathrm{~B} ; 7 \mathrm{~min}, 65 \% \mathrm{~B} ; 8-9 \mathrm{~min}, 40 \% \mathrm{~B}$; 9.1-12 $\mathrm{min}, 95 \% \mathrm{~B}$. The flow rate was set to $0.5 \mathrm{~mL} / \mathrm{min}$. The injection volume of the test sample was $1 \mu \mathrm{L}$.

The mass spectrometer was operated using TripleTOF6600 triple four-pole time-of-flight mass spectrometry (ABSciex, America). Collision energy (CE) was $30 \mathrm{eV}$. The parameters of the electron spray ionization (ESI) source were set as follows: ion source gas 1, 60 psi; ion source gas 2, $60 \mathrm{psi}$; source temperature, $600{ }^{\circ} \mathrm{C}$; and ion spray voltage floating (ISVF), $5 \mathrm{kV}$ or $-4 \mathrm{kV}$ in positive or negative mode, respectively.

\section{Data processing and multivariate data analysis}

The mass spectrometer original data from the LC-MS analysis of the metabolites were converted to the common (mzXML) format by ProteoWizard. Then, the converted data were imported into XCMS 3.2 software (http://bioconductor.org/packages/release/bioc/html/ $\mathrm{xcms} . h t m l)$ to generate a data matrix that involved the normalized peak intensity, exact mass, and retention time. These metabolome data were input into the metaboanalyst 4.0 platform (https://www.metaboanalyst.ca) for multivariate statistical analysis. Principal component analysis (PCA) and a partial least squares-discriminant analysis (PLS-DA) model constructed by the pattern recognition method were used to identify the underlying difference in the metabolites between the groups with different inflammatory grades.

\section{Microbiome analysis Microorganism extraction}

A 30-mg piece of periapical specimen in an Eppendorf tube was treated for total genomic DNA extraction according to the manufacturer's instructions (E.Z.N.A. ${ }^{\circledR}$ Stool DNA Kit, Omega Bio-tek, Norcross, GA, U.S.). The yield and purity of the DNA were measured using a Nanodrop 8000 (Thermo Scientific, Wilmington, DE, USA). DNA was used to study microbial diversity by amplifying the V3-V4 region of the $16 \mathrm{~S}$ rRNA gene using the universal primers $338 \mathrm{~F}$ (5'-GTACTCCTACGGGAGGCA GCA-3') and 806R (5'-GTGGACTACHVGGGTWT CTAAT- $3^{\prime}$ ). The PCR mixture contained $30 \mathrm{ng}$ template DNA, $1 \mu \mathrm{L}$ upstream primer $(5 \mu \mathrm{mol} / \mathrm{L}), 1 \mu \mathrm{L}$ downstream primer $(5 \mu \mathrm{mol} / \mathrm{L}), 1 \mu \mathrm{L}$ BSA $(1 \mathrm{ng} / \mu \mathrm{L})$, and 12.5 $\mu \mathrm{L} 2 \times$ Taq Plus Master Mix, and sterile Milli-Q water was used to adjust the final volume to $25 \mu \mathrm{L}$. PCR was performed with an initial denaturation at $94{ }^{\circ} \mathrm{C}$ for $5 \mathrm{~min}, 45$ cycles of denaturation at $94{ }^{\circ} \mathrm{C}$ for $30 \mathrm{~s}$, annealing at $50{ }^{\circ} \mathrm{C}$ for $30 \mathrm{~s}$, and extension at $72{ }^{\circ} \mathrm{C}$ for $60 \mathrm{~s}$, and a final extension at $72{ }^{\circ} \mathrm{C}$ for $10 \mathrm{~min}$. Post-amplification quality control was performed by electrophoresis with a $2 \%$ agarose gel. PCR products were purified with AMPure XP Beads (Beckman Coulter, Milan, Italy).

\section{Data analysis}

The microbiome data acquired from the $16 \mathrm{~S}$ rRNA highthroughput sequencing were imported and analysed by QIIME 1.8.0 software (http://qiime.org/). OTUs (operational taxonomic units) were picked at $99 \%$ similarity with UCLUST clustering methods to predict the abundances of species based on OTU data according to the Silva databases. 


\section{Statistical analysis}

The metabolites with a variable importance in project (VIP) $>1$ in the PLS-DA model and a $p$ value $<0.05$ in the analysis of variance were considered potential biomarkers identified by the LC-MS analysis. The affected metabolic pathways were explored via Metabolomic Pathway Analysis software (https://www.metaboanalyst. ca), and those with an impact value $>0.1$ and a $p$ value $<0.05$ were selected as the significantly different pathways. Analysis of variance was conducted to compare the relative abundance of major microorganisms associated with periapical periodontitis with different inflammation grades, and all the data were analysed with SPSS 17.0 software (Chicago, USA). Significant differences between the metabolome and microbiome of periapical periodontitis were assessed using Spearman's correlation analysis. The $\mathrm{R}$ language (pheatmap package) was used to analyse the Spearman correlation coefficient, and the resulting numerical matrix was displayed using a heat map.

\section{Results}

\section{Haematoxylin and eosin (H\&E) staining and inflammatory} grade

Among 47 specimens, the histopathological examination showed 11 specimens (23.4\%) with slight inflammation (grade I), 20 specimens $(42.6 \%)$ with moderate inflammation (grade II), and 16 specimens (34.0\%) with severe inflammation (grade III). The lesions of periapical periodontitis in the sections stained with haematoxylin and eosin (H\&E) included immune cells (dominantly macrophages and lymphocytes), proliferative capillaries, and fibrous tissue (Figs. 1A, 2A, 3A). After light microscopic examination, the lesions of the periapical tissues were divided into three grades based on inflammatory cell infiltration. Then, the samples analysed to determine the relationship of the local metabolome and the type and abundance of microorganisms were subsequently divided into three groups based on the different inflammatory grades: grade I, grade II, and grade III (Figs. 1, 2, 3). Figures 1, 2 and 3 show examples of histopathology and $\mathrm{CBCT}$ of the periapical periodontitis specimens.

\section{Metabolome analysis of periapical periodontitis Metabolome analysis}

In the PCA score plot (Fig. 4A, B), the QC samples (light blue scatters) were clustered together in the centre of the plot (inside a small light blue circle in Fig. 4A, B, respectively), indicating satisfactory stability regarding sample quality and analytical methods [13]. The samples inside the red, green, and purple circles represent the metabolic profiles of periapical periodontitis with grade I, grade II and grade III, respectively. Moreover, the graph of the
PCA statistical analysis model of periapical periodontitis also showed that the periapical periodontitis samples in the same group were clustered in one area (inside the red, green and purple circles of Fig. 4A, B, respectively), meaning that there was similarity in the main component in the samples with the same inflammatory grades (Fig. 4A, B). The metabolic profiles in the graph of the PCA model from of periapical periodontitis with different inflammatory grades were obviously separated and different in both negative and positive ion modes (red, green, and purple circle of Fig. 4A, B), which suggested that metabolic differences existed among three different inflammatory grades.

The PLS-DA score plots (Fig. 5C, D) revealed that there was a clear separation in the different grades of periapical periodontitis, while less separation was observed within any particular grade (Fig. 5A, B). This result indicated that there was a significant intergroup difference among the different inflammatory grades. The development of periapical periodontitis may be associated with important metabolic disorders and pathological physiological changes. Of note, the three different groups of periapical periodontitis partly overlapped (Fig. 5A, B), which suggested that the changes in inflammation were gradual and continuous.

\section{Metabolite identification}

Eight potential biomarkers, such as $\mathrm{N}$-acetyl-D-glucosamine, were identified in the three periapical periodontitis groups by LC-MS analysis in cation mode (Table 1), while ten potential biomarkers, such as L-phenylalanine, were obtained in anion mode (Table 2). These differential metabolites identified above were involved in changes in amino acid metabolism, sugar metabolism, and fatty acid metabolism during the progression of periapical periodontitis. The potential and possible roles of metabolites in periapical periodontitis are shown in Tables 1 and 2 . Then, a heat map was constructed to visualize the expression of these differential metabolites in the different periapical periodontitis groups (Fig. 6). The heat map showed that the colour of the metabolites in the grade I and II groups only slightly changed, while that in the grade III group obviously changed. This result indicated that the expression of the metabolites associated with periapical periodontitis increased more obviously in the grade III group than in the grade I and II groups.

\section{Metabolomic pathway interpretation}

The identified biomarkers responsible for periapical periodontitis were further analysed by MetaboAnalyst 4.0 (https://www.metaboanalyst.ca/). The pathway analysis (Fig. 7) showed that the 18 potential biomarkers of periapical lesions were mapped to 26 pathways. Six pathways 

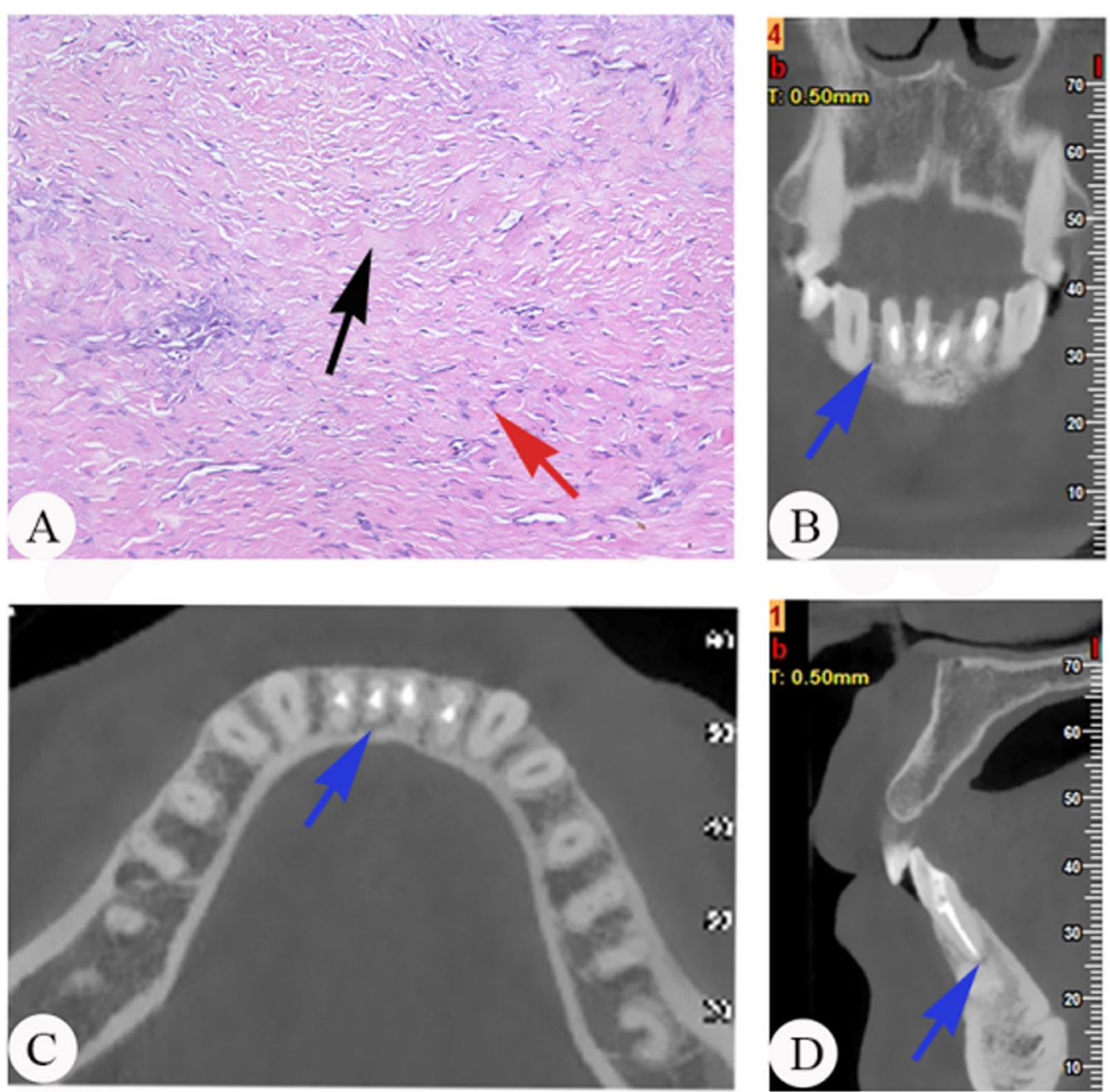

Fig. 1 Examples of included cases with histopathology and CBCT sections. A Histopathology of periapical lesions ( $\times 200$, H\&E staining) in grade I. Fibrous/scar tissue with slight inflammation. The immune cells (red arrow) and proliferative fibrous tissue (black arrow) exist in the periapical tissue. B CBCT, coronal section of periapical tissue, blue arrow points at periapical lesions. C CBCT, horizontal section of periapical tissue, blue arrow points at periapical lesions. D CBCT, sagittal section of periapical tissue, blue arrow points at periapical lesions

with an impact value $>0.1$ and a $p$ value $<0.05$ were selected as the significantly different pathways in the comparison between grades I and III and grades II and III. Among the six metabolic pathways, five pathways were associated with amino acid metabolism, including aminoacyl-tRNA biosynthetic, phenylalanine, tyrosine, and tryptophan biosynthesis; phenylalanine metabolism; alanine, aspartate, and glutamate metabolism; and cysteine and methionine metabolism; one pathway was related to sugar metabolism. Moreover, 13 metabolites were correlated with the six metabolic pathways mentioned above (Table 3).

Figure 8 shows the relative intensity of 13 key metabolites in the samples of periapical periodontitis with different inflammatory grades. The relative concentrations of
13 metabolites in the grade III periapical periodontitis group were significantly increased compared with those in the grade I and grade II periapical periodontitis group $(p<0.05)$. However, there was no significant difference in the relative concentrations of 13 metabolites between the grade I and II periapical periodontitis groups $(p>0.05)$.

The metabolic networks revealed potential connections between the 13 key biomarkers and the relevant metabolic pathways in periapical periodontitis (Fig. 9). Asparagine of the alanine metabolic pathway was potentially regulated by or connected to the phenylalanine, tyrosine and tryptophan biosynthesis pathways; aspartate of the aspartate metabolic pathway was potentially regulated by or connected to the cysteine and tryptophan metabolic pathways; and glutamate of the glutamate metabolic 

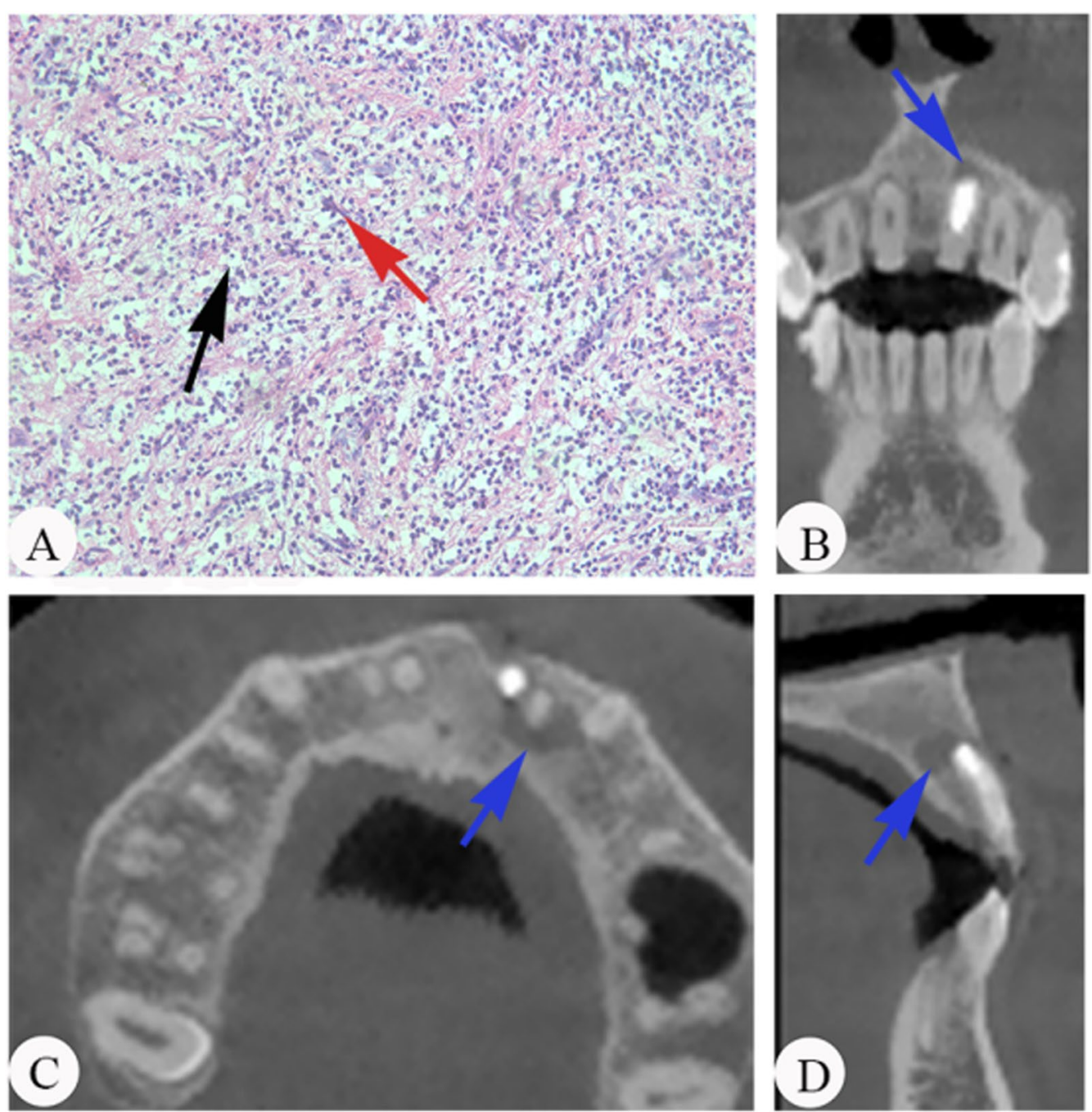

Fig. 2 Examples of included cases with histopathology and CBCT sections. A Histopathology of periapical lesions $(\times 200$, H\&E staining) in grade II. Fibrous/scar tissue with moderate inflammation. The immune cells (red arrow) and proliferative fibrous tissue (black arrow) exist in the periapical tissue. B CBCT, coronal section of periapical tissue, blue arrow points at periapical lesions. C CBCT, horizontal section of periapical tissue, blue arrow points at periapical lesions. D CBCT, sagittal section of periapical tissue, blue arrow points at periapical lesions

pathway was potentially regulated by or connected to the amino sugar and nucleotide metabolic pathways. Compared to those associated with lower degrees of inflammation, the key biomarkers in these metabolic pathways associated with higher degrees of inflammation were significantly increased in periapical periodontitis $(p<0.05)$ (Fig. 9).

\section{Microbiome analysis of periapical periodontitis}

After importing $16 \mathrm{~S}$ rRNA high-throughput sequencing data into QIIME 1.8.0 software, the data showed that the bacterial populations associated with periapical periodontitis were characterized by the presence of Porphyromonas, Fusobacterium, Streptococcus, Bacillus, and Actinomycetes.
Regardless of the severity of inflammation, the proportions of microorganisms, in a sequence from higher to lower, were Porphyromonas, Fusobacterium, and Streptococcus. There was no significant correlation between the inflammatory grade and the type or abundance of infectious microorganisms associated with periapical periodontitis using variance analysis (all $p>0.05$ ). Representative microorganisms of periapical periodontitis with different inflammatory grades are presented in Fig. 10.

\section{Correlation between the metabolome and microbiome of periapical periodontitis}

The correlation between thirteen key metabolites and the type or relative abundance of ten major microorganisms 

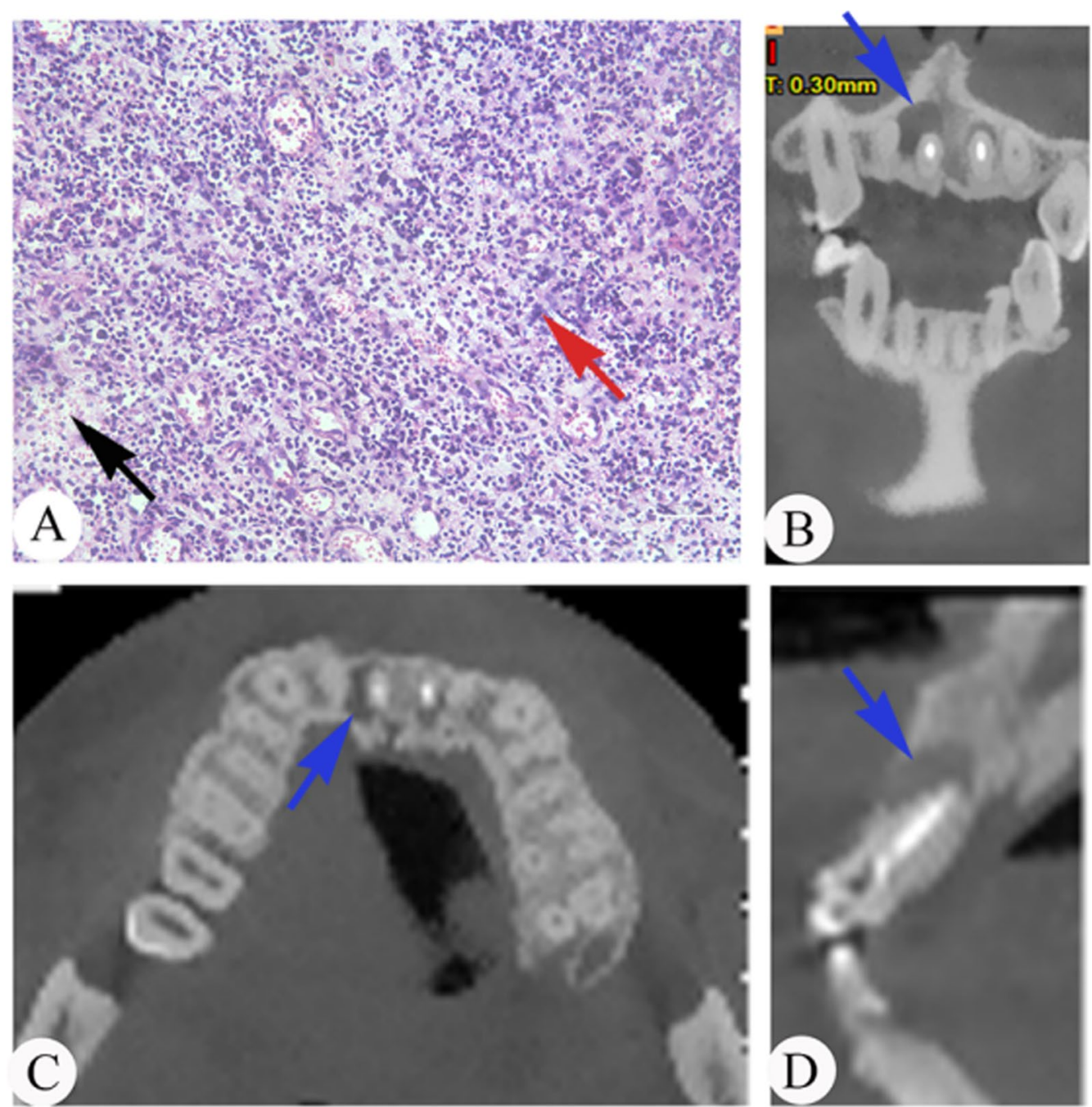

Fig. 3 Examples of included cases with histopathology and CBCT sections. A Histopathology of periapical lesions ( $\times 200$, H\&E staining) in grade III. Fibrous/scar tissue with severe inflammation. The immune cells (red arrow) and proliferative fibrous tissue (black arrow) exist in the periapical tissue. B CBCT, coronal section of periapical tissue, blue arrow points at periapical lesions. C CBCT, horizontal section of periapical tissue, blue arrow points at periapical lesions. D CBCT, sagittal section of periapical tissue, blue arrow points at periapical lesions.

was further analysed (Fig. 11). The abundance of Actinomycetes was negatively correlated with the abundance of glucosamine (GlcN), while the abundance of Tannerella was positively correlated with the abundance of L-methionine.

\section{Discussion}

In recent years, metabolome or microbiome analysis techniques have emerged as objective tools for identifying the metabolomic mechanisms underlying the development of chronic inflammatory disorders mainly caused by bacterial infection. A microbiome-metabolome study revealed that the gut microbiota was associated with glycine-conjugated metabolites and polyamine metabolism in chronic kidney disease [26]. Recently, metabolomics of oral diseases has made certain progress. Gokul analysed and identified serum metabolites in oral leukoplakia and oral squamous cell carcinoma using quadrupole time of flight-liquid chromatography-mass spectrometry [27], and Barnes performed an unbiased metabolomic profiling of gingival crevicular fluid (GCF) collected from healthy, gingivitis and periodontitis sites in humans using liquid and gas chromatography mass spectrometry [28]. However, the local microbiome-metabolome alterations that occur in the development of periapical periodontitis have not been discussed in previous studies.

In this study, the levels of phenylalanine, tyrosine, and tryptophan in the phenylalanine metabolism pathway 

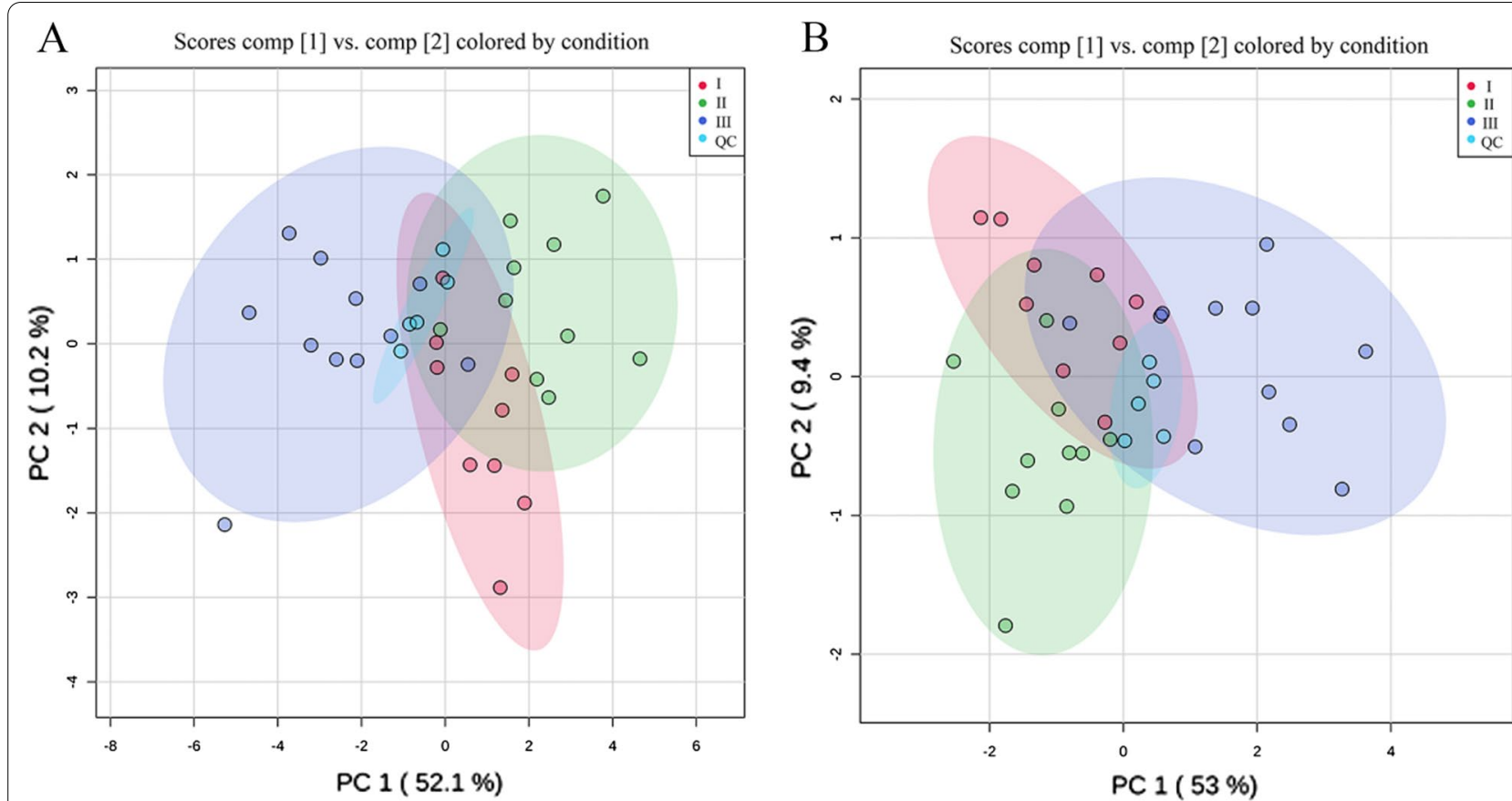

Fig. 4 PCA statistical analysis model of periapical periodontitis using UPLC-QTOF/MS. The abscissa represents the first principal component and the ordinate represents the second principal component. A Positive ion mode, $\mathbf{B}$ negative ion mode. The red, green, and purple scatters represent the metabolic profiles of periapical periodontitis as grade I, grade II, and grade III, respectively. Light blue scatters represent the QC samples. Each scatters represents a sample, and the closer the scatter is, the higher the similarity of the principal components of the samples exists

increased significantly in the grade III periapical periodontitis samples compared with the grades I and II periapical periodontitis samples. High levels of phenylalanine inhibit the activity of 5-hydroxytryptophan decarboxylase and decrease the synthesis of 5-hydroxytryptamine (5-HT) from tryptophan [17]. Moreover, tyrosine is a nonessential amino acid that is synthesized from phenylalanine by phenylalanine hydroxylase. This result suggested that the high level of phenylalanine might be positively correlated with the increase in tryptophan and tyrosine levels. A previous study demonstrated that tryptophan has strong anti-inflammatory activity, which might be explained by its significant inhibitory effect on IL-8 secretion by interfering with TNF- $\alpha$-activated inflammatory signal transduction [23]. In this study, the overproduction of phenylalanine in periapical periodontitis might subsequently increase tryptophan production. Therefore, hyperactivity of tryptophan in periapical periodontitis might decrease the production of local proinflammatory factors and increase the local anti-inflammatory activity to physically protect against bacterial inflammation.

The levels of alanine, glutamine and asparagine in the alanine, aspartate and glutamate metabolic pathways were increased in grade III periapical periodontitis samples compared with the grade I and II periapical periodontitis samples. Alanine and asparagine play central roles in maintaining metabolism, protein synthesis, nitrogen balance and immune responses in cells [20]. Moreover, alanine, glutamine and asparagine have anti-inflammatory activities that are related to TNF- $\alpha$ and IL-1 $\beta$, which can activate osteoclasts and stimulate bone resorption [18]. Therefore, the increase in alanine, glutamine and asparagine levels in periapical periodontitis might suppress the production of proinflammatory cytokines and the expansion of inflammation in periapical periodontitis.

The levels of methionine, serine, and 3-methylthiopropionate in the cysteine and methionine metabolic pathways were increased obviously in the grade III periapical periodontitis samples compared with the grade I and II periapical periodontitis samples. Methionine has important antioxidant and antiresorptive effects by inhibiting the propensity of mononuclear cells to develop into functional osteoclasts. 3-Methylthiopropionate is an intermediate in the methionine catabolism pathway [15]. Therefore, elevated levels of methionine and 3-methylthiopropionate might regulate the local production of proinflammatory cytokines, suppress the differentiation of osteoclasts, and attenuate bone loss in periapical periodontitis. In addition, serine is an essential metabolite for regulating clonal $\mathrm{T}$ lymphocyte expansion in adaptive 
A

Scores comp [1] vs. comp [2] colored by condition

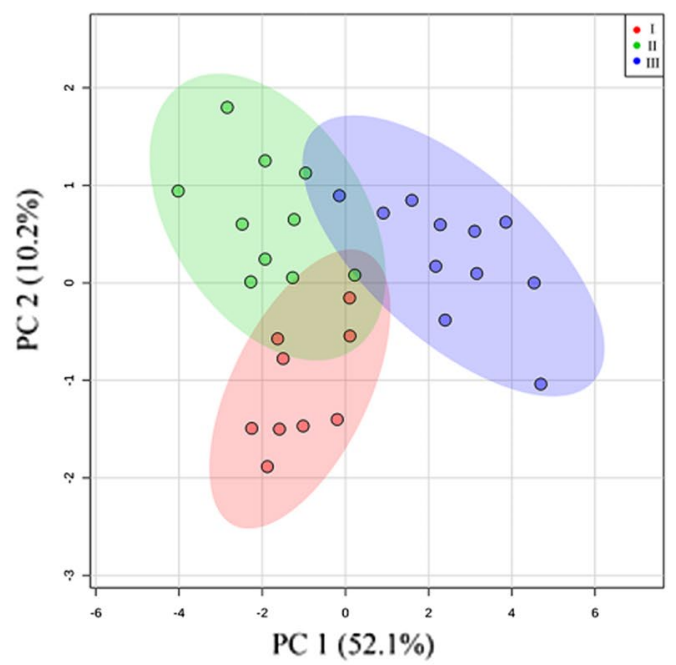

C

Scores comp [1] vs. comp [2] vs. comp [3] colored by condition

$t(2)$

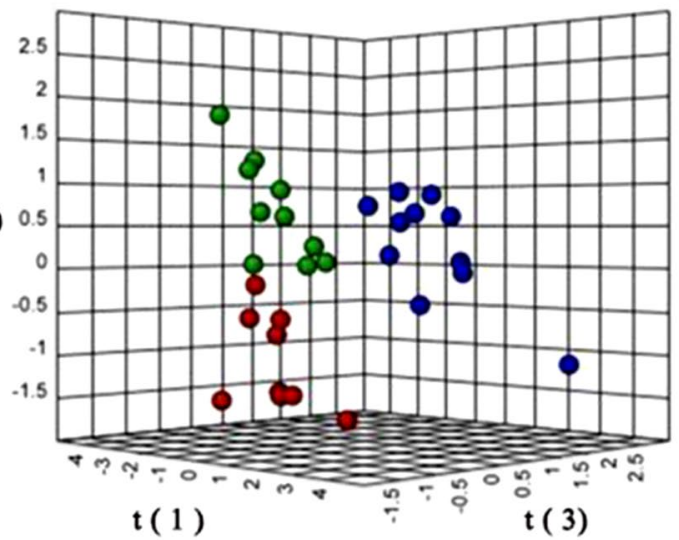

B

Scores comp [1] vs. comp [2] colored by condition

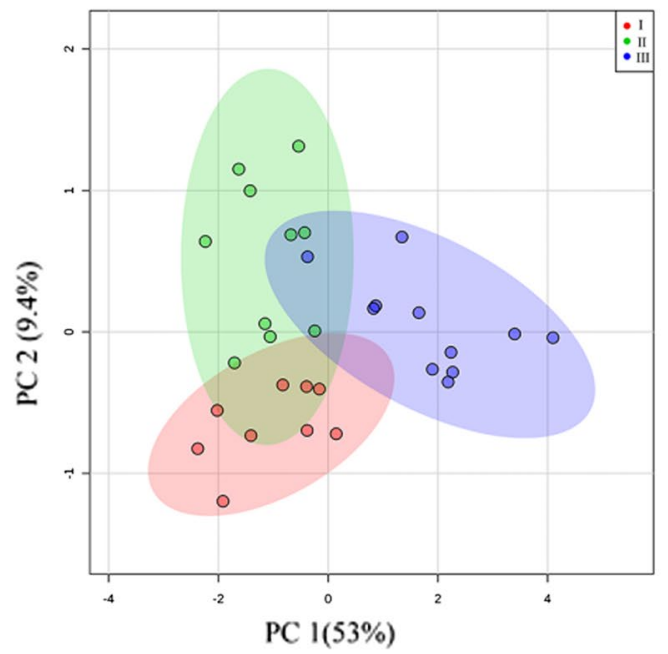

$\mathrm{D}$

Scores comp [1] vs. comp [2] vs. comp [3] colored by condition

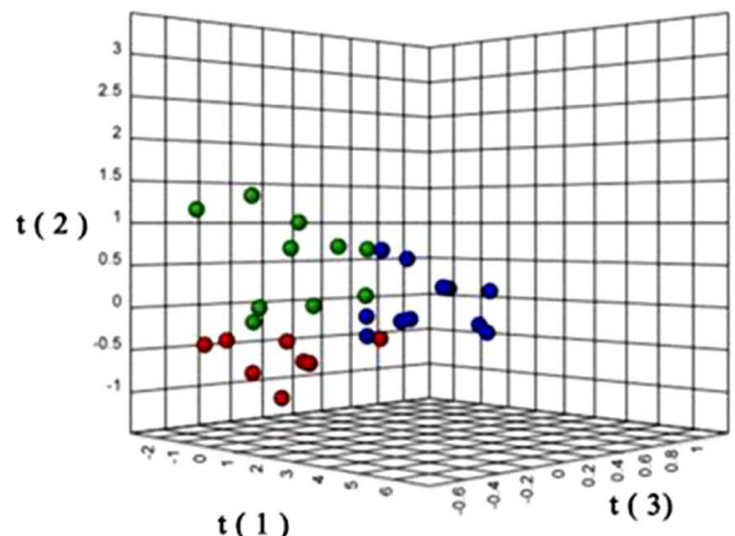

Fig. 5 PLS-DA and 3D PLS-DA statistical analysis models of periapical periodontitis. A PLS-DA statistical analysis models of periapical periodontitis in positive ion mode; $\mathbf{B}$ PLS-DA statistical analysis models of periapical periodontitis in negative ion mode; C 3D PLS-DA statistical analysis models of periapical periodontitis in positive ion mode; D 3D PLS-DA statistical analysis models of periapical periodontitis in negative ion mode. Red, green, and purple spots respectively represent the samples of periapical periodontitis at grade I, grade II, and grade III. Each scatters represents a sample. The closer the scatter is, the smaller of the difference between the samples exists

Table 1 Potential bio-markers of periapical lesions in cation mode

\begin{tabular}{lllll}
\hline Name & VIP & P value & Potential role & References \\
\hline N-Acetylmannosamine & 2.5741 & $3.16 \mathrm{E}-09$ & & Anti-inflammatory, antioxidant \\
N-acetyl-D-glucosamine & 2.0183 & $2.18 \mathrm{E}-07$ & Methionine metabolites & [14] \\
3-methylthiopropionate & 1.9203 & $1.49 \mathrm{E}-07$ & Improving the immune function & \\
Tyramine & 1.423 & $6.06 \mathrm{E}-05$ & & \\
Benzene lactic acid & 1.3575 & 0.00013 & & \\
L-Tyrosine & 1.2729 & $3.35 \mathrm{E}-05$ & Anti-inflammatorry,Inhibiting osteoclast \\
Thymine & 1.2627 & $2.77 \mathrm{E}-05$ & $7.25 \mathrm{E}-06$ & [15] \\
L-Methionine & 1.2441 & &
\end{tabular}


Table 2 Potential bio-markers of periapical lesions in anion mode

\begin{tabular}{|c|c|c|c|c|}
\hline Name & VIP & $P$ value & Potential Role & References \\
\hline L-Phenylalanine & 1.7786 & $6.02 \mathrm{E}-06$ & Inhibiting 5-phenylalanine decarboxylase & [17] \\
\hline L-Glutamate & 1.5181 & 0.000137 & Increasing IFN- $\gamma$ and IL-10 & [18] \\
\hline L-Tryptophan & 1.5134 & $3.79 \mathrm{E}-06$ & Decreasing $\|-1 \beta\|-6,, \mid L-8$, TNF- $\alpha$ & [19] \\
\hline L-Alanine & 1.4168 & $1.44 \mathrm{E}-05$ & Decreasing TNF-a & {$[20]$} \\
\hline Glucosamine & 1.3915 & 0.000693 & anti-inflammary, antioxidant & {$[14,19]$} \\
\hline L-Serine & 1.3393 & $4.59 \mathrm{E}-05$ & Regulating of T-cell Proliferation & {$[21]$} \\
\hline L-Asparagine & 1.2961 & $1.93 \mathrm{E}-05$ & Decreasing TNF- $\alpha$ and IL-1 $\beta$ & [22] \\
\hline L-Valine & 1.1814 & $2.12 \mathrm{E}-06$ & $\begin{array}{l}\text { Generating immunoglobulin } \\
\text { Decreasing TNF- } a, \mathrm{IL}-6, \mathrm{INF}-\gamma, \mathrm{IL}-1 \beta \text { and IL-17 }\end{array}$ & [23] \\
\hline Linoleic acid & 1.1752 & 0.011785 & No influence & [24] \\
\hline Taurine & 1.0241 & 0.013787 & Decreasing TNF-a, IL-6 and Oxidative stress & {$[25]$} \\
\hline
\end{tabular}

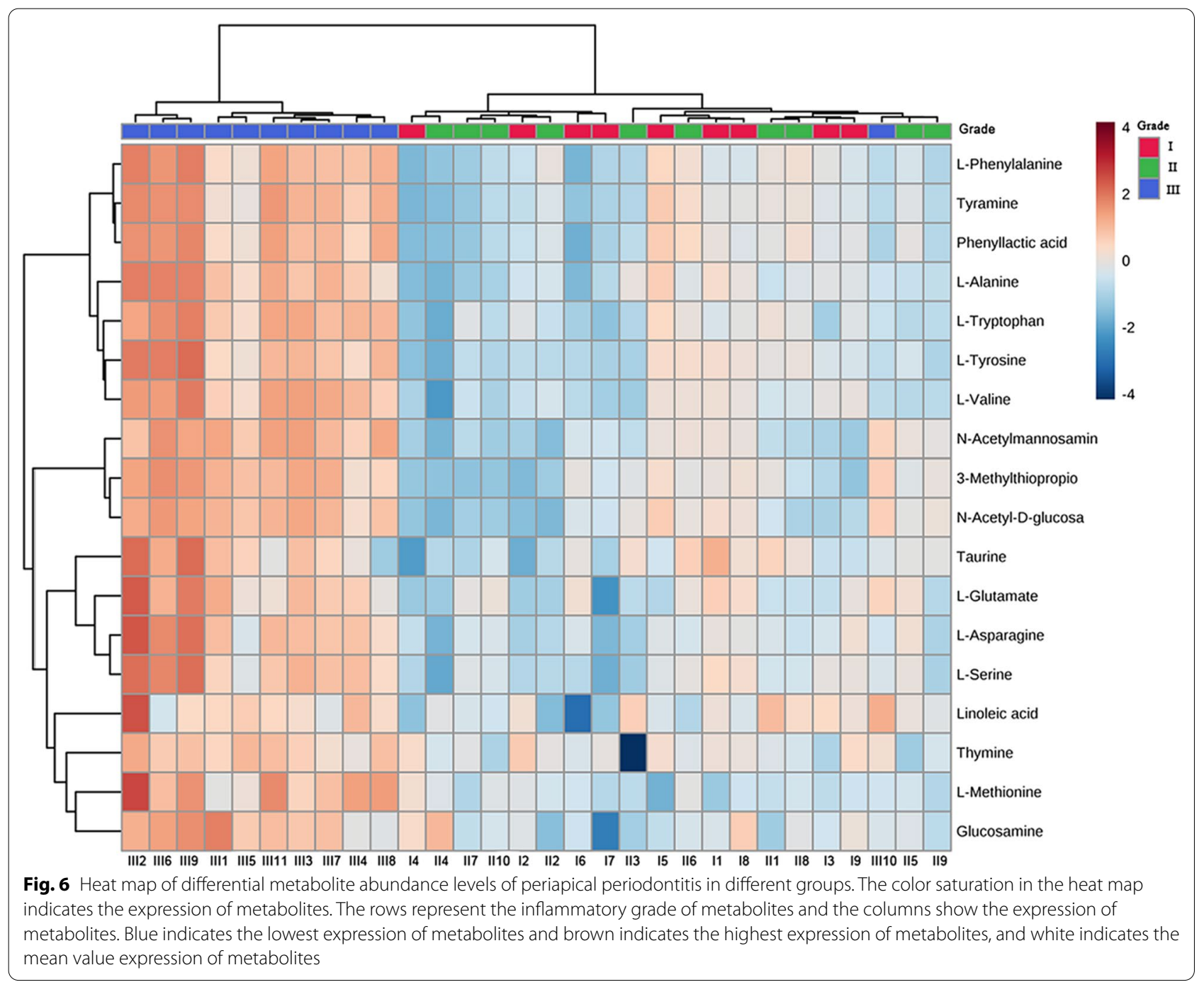




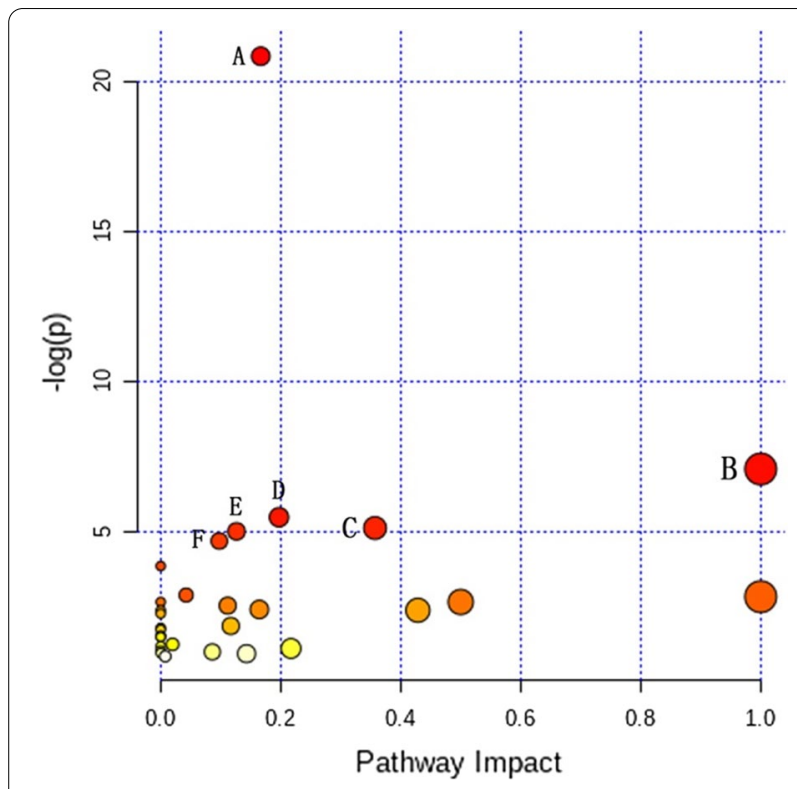

Fig. 7 The metabolome view map of relevant metabolic pathways in periapical periodontitis metabolic profiles. The ordinate with $p$ value (take the negative common logarithm, that is, $-\log _{10} p$ ) is calculated from the enrichment analysis; the abscissa with an impact value of the pathway is obtained from topology analysis. Each bubbles represents a metabolic pathway of the sample. Regarding the ordinate, the higher the $-\log _{10} p$ of the bubble is, the smaller the $p$ value of the sample is. Regarding the abscissa, the bigger the size of the bubble is, the greater the impact value of the sample is. A Aminoacyl-tRNA biosynthetic pathway; B Phenylalanine, tyrosine and tryptophan biosynthesis pathway; C Phenylalanine metabolism pathway; D Alanine, aspartate and glutamate metabolism pathway; E Cysteine and methionine metabolism pathway; F Amino sugar and nucleotide sugar pathway immunity [21]. Therefore, the increased expression of serine in periapical periodontitis could be associated with autoimmune regulation in periapical periodontitis.

The levels of glucosamine (GlcN), N-acetylmannosamine (ManNAc), and N-acetyl-D-glucosamine (GlcNAc) in the cysteine and methionine metabolic pathways were increased significantly in the grade III periapical periodontitis samples compared with the grade I and II periapical periodontitis samples. ManNAc is the metabolite of GlcN and GlcNAc. Both GlcN and GlcNAc attenuate IL-1 $\beta$, IL-6, and TNF- $\alpha$ expression in normal human articular chondrocytes, and GlcN plays a novel role in the alleviation of oxidative stress and lung inflammation in rats [14]. These findings indicate that GlcN and GlcNAc might directly reduce local inflammation in periapical periodontitis by inhibiting anti-inflammatory cytokine production and alleviating oxidative stress.

The microbial community associated with in periapical periodontitis is complex and diverse. In this study, the severity of inflammation had no obvious correlation with the abundance of single microorganisms, suggesting that persistent periapical periodontitis is related to combined polymicrobial infections. Gomes et al. investigated the presence of nine bacterial species in root-filled teeth associated with periapical lesions using the same method and found that Enterococcus faecalis, Porphyromonas gingivalis and Peptostreptococcus micros were the most frequently identified species [1]. Federico Mussano et al. found that facultative anaerobes were dominant in periapical granulomas and radicular cysts, but the anaerobic types were the most abundant in radicular cyst samples [29]. In this study, Porphyromonas, Fusobacterium, and Streptococcus were the predominant species in all three

Table 3 Metabolite changes in the metabolic pathway analysis of periapical periodontitis

\begin{tabular}{|c|c|c|c|c|c|c|}
\hline & Metabolic pathway & $\begin{array}{l}\text { The total } \\
\text { number of } \\
\text { metabolites }\end{array}$ & $\begin{array}{l}\text { The total } \\
\text { number of } \\
\text { differential } \\
\text { metabolites }\end{array}$ & Metabolites & $P$ value & $-\log (p)$ value \\
\hline 1 & Aminoacyl-tRNA biosynthesis & 48 & 9 & $\begin{array}{l}\text { L-Asparagine, L-Phenylalanine, } \\
\text { L-Serine, L-Methionine, } \\
\text { L-Valine, L-Alanine, } \\
\text { L-Tryptophan, L-Tyrosine, } \\
\text { L-Glutamine }\end{array}$ & 0.00 & 21.47 \\
\hline 2 & $\begin{array}{l}\text { Phenylalanine, tyrosine and tryptophan biosyn- } \\
\text { thesis }\end{array}$ & 4 & 2 & $\begin{array}{l}\text { L-Phenylalanine, } \\
\text { L-Phenylalanine }\end{array}$ & 0.00 & 7.19 \\
\hline 3 & Alanine, aspartate and glutamate metabolism & 28 & 3 & $\begin{array}{l}\text { L-Asparagine, } \\
\text { L-Alanine, L-Glutamate }\end{array}$ & 0.00 & 5.63 \\
\hline 4 & Phenylalanine metabolism & 10 & 2 & $\begin{array}{l}\text { L-Phenylalanine, } \\
\text { L-Tyrosine }\end{array}$ & 0.01 & 5.22 \\
\hline 5 & Cysteine and methionine metabolism & 33 & 3 & $\begin{array}{l}\text { L-Serine, } \\
\text { L-Methionine, } \\
\text { 3-methylthiopropionate }\end{array}$ & 0.01 & 5.16 \\
\hline 6 & Amino sugar and nucleotide sugar metabolism & 37 & 3 & $\begin{array}{l}\mathrm{N} \text {-Acetylmannosamine, } \\
\mathrm{N} \text {-acetyl-D-glucosamine }\end{array}$ & 0.01 & 4.83 \\
\hline
\end{tabular}



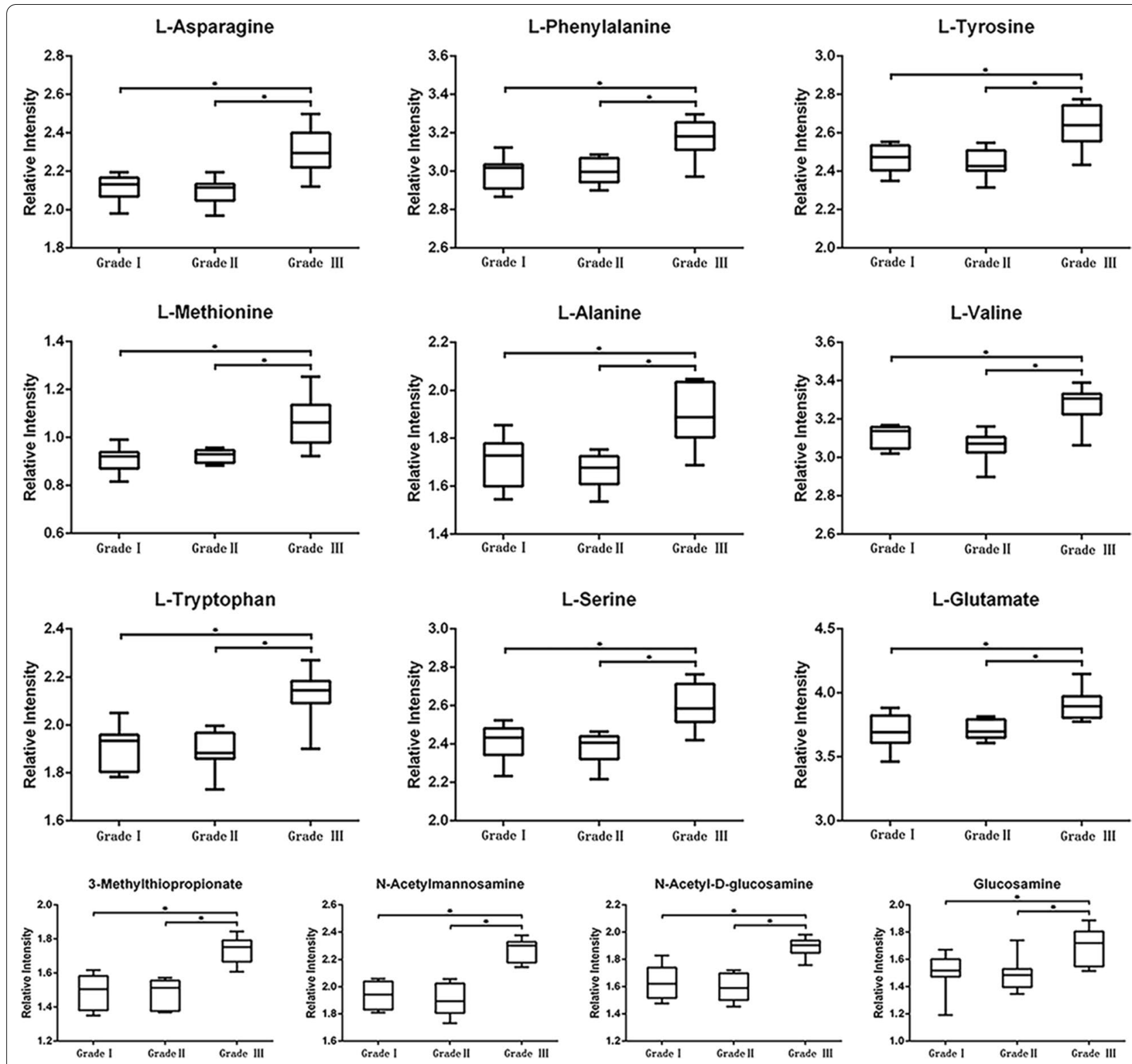

L-Serine

L-Glutamate
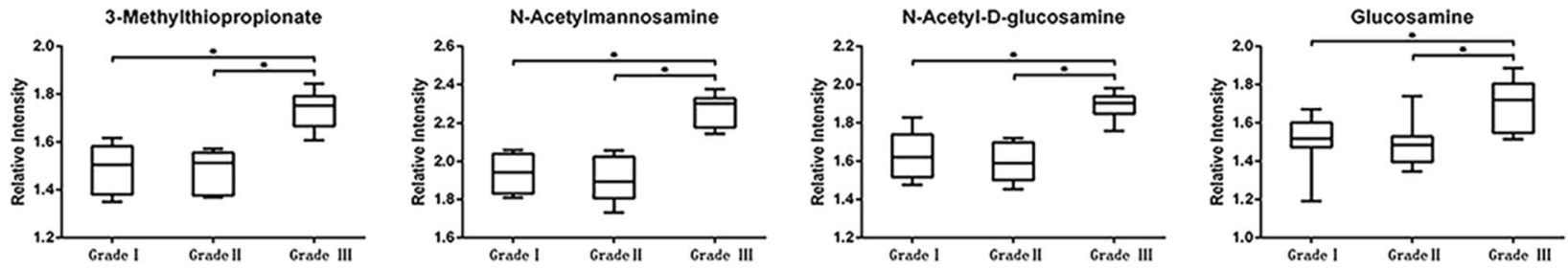

Fig. 8 Changes in relative concentrations of 13 key metabolites in different immune cell infiltration levels $\left({ }^{*} p<0.05\right)$

groups of periapical periodontitis samples, regardless of the severity of inflammation. The difference in the microbial species may be attributed to the difference in human ethnicities, individual immune systems, clinical materials, tissue location, sample size and treatment procedures [30].

In this study, the abundance of Actinomycetes was negatively correlated with the abundance of glucosamine $(\mathrm{GlcN})$. Studies show that GlcN has immune regulatory functions and regulates the activation of IL-1 $\beta$
[14]. Actinomyces has been commonly found in primary root canal infections and is also known as an important microorganism in the development of persistent apical periodontitis lesions [31]. Further study showed that GlcN is positively related to amino sugar metabolism in Actinomycetes, and it functions by enhancing the role of glycogen and improving the catalytic performance. In addition, the abundance of Tannerella was positively correlated with that of L-methionine. L-Methionine inhibits the transdifferentiation of osteoclasts and the synthesis of proinflammatory cytokines, such as IL- $1 \alpha$ 


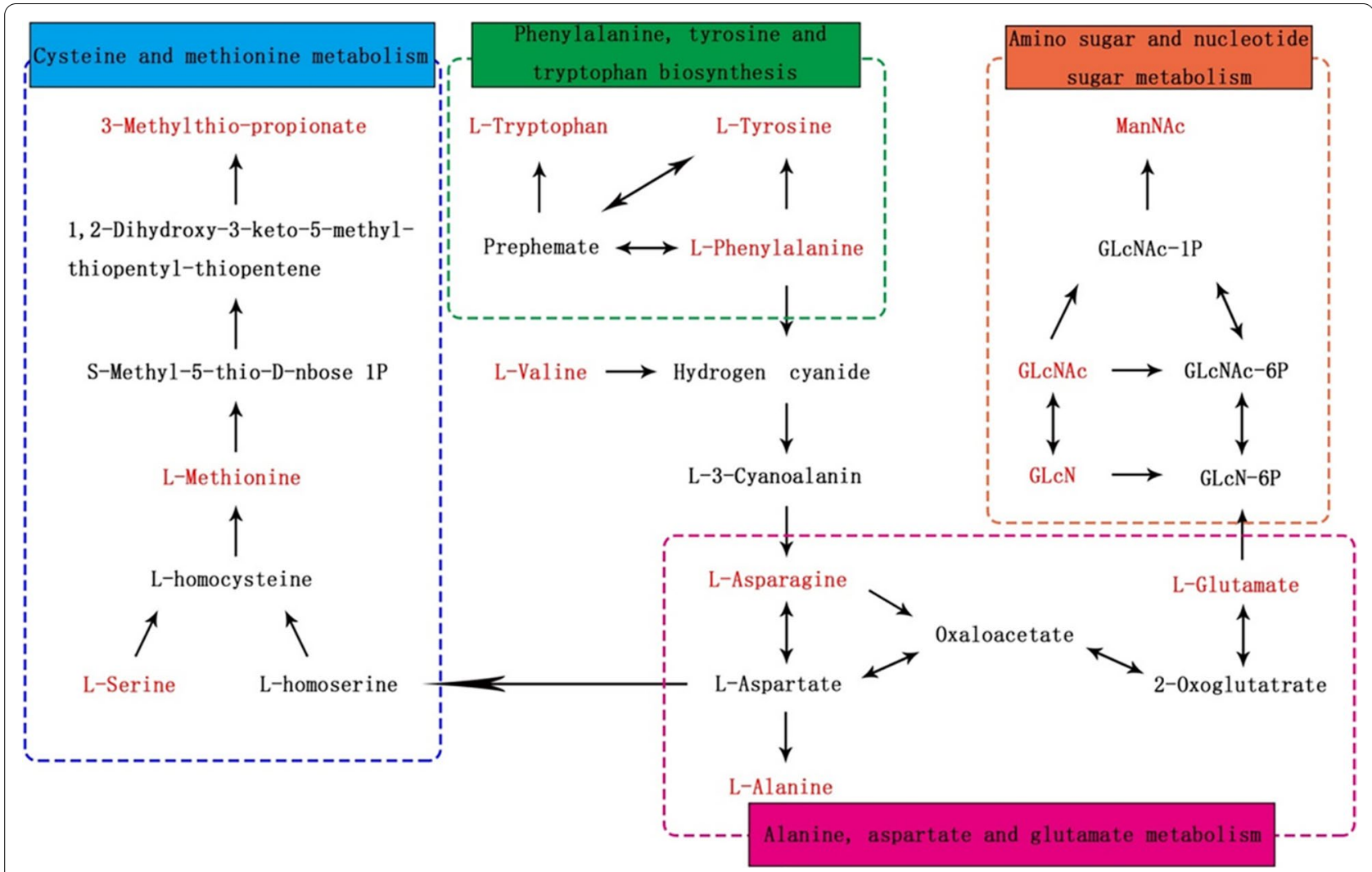

Fig. 9 Key biomarkers and the relevant metabolic networks identified from the KEGG database. Red words represent 13 key biomarkers in periapical periodontitis and indicate the increased expression of metabolites around the periapical lesions

[15]. Tannerella is one of the prevalent microorganisms in both primary periapical periodontitis and endodontic failure and can induce the activation of inflammasomes in periapical periodontitis [32]. Tannerella might

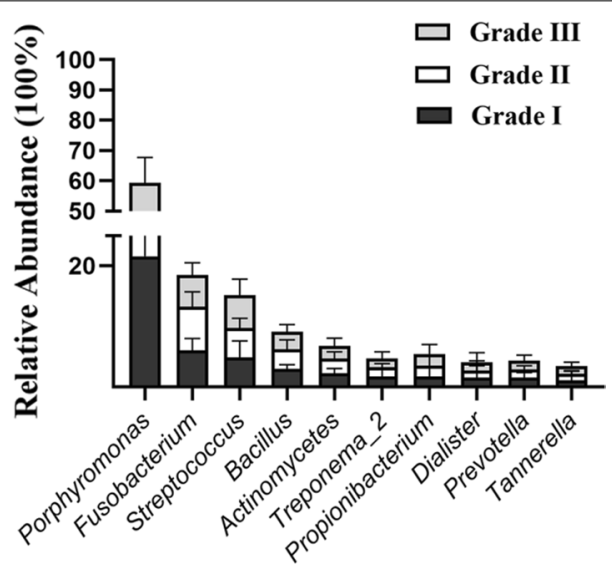

Fig. 10 Comparisons of relative abundance of ten major microorganisms from periapical periodontitis with different inflammation degrees. $p$ values are based on variance analysis and all $p>0.05$ increase the inflammatory grade by inhibiting the metabolism of L-methionine in periapical periodontitis; however, there are still no studies on the correlation between Tannerella and L-methionine, which needs to be discussed in the future.

Here, we investigated the relationship among local metabolome alterations, inflammatory grades, and types and abundances of microorganisms associated with periapical periodontitis. This study found that periapical periodontitis was related to changes in amino acid, sugar and fatty acid metabolism, and the more severe the inflammation was, the more obvious the metabolic changes were. This finding indicates that the local immune system and metabolism are involved in the occurrence and development of periapical periodontitis. Additionally, metabolites have strong antiinflammatory activity and can inhibit the production of osteoclasts. The increased levels of these metabolites suggest that physiological regulation occurs during the development of periapical periodontitis. The mechanisms underlying this observation need to be further studied. 


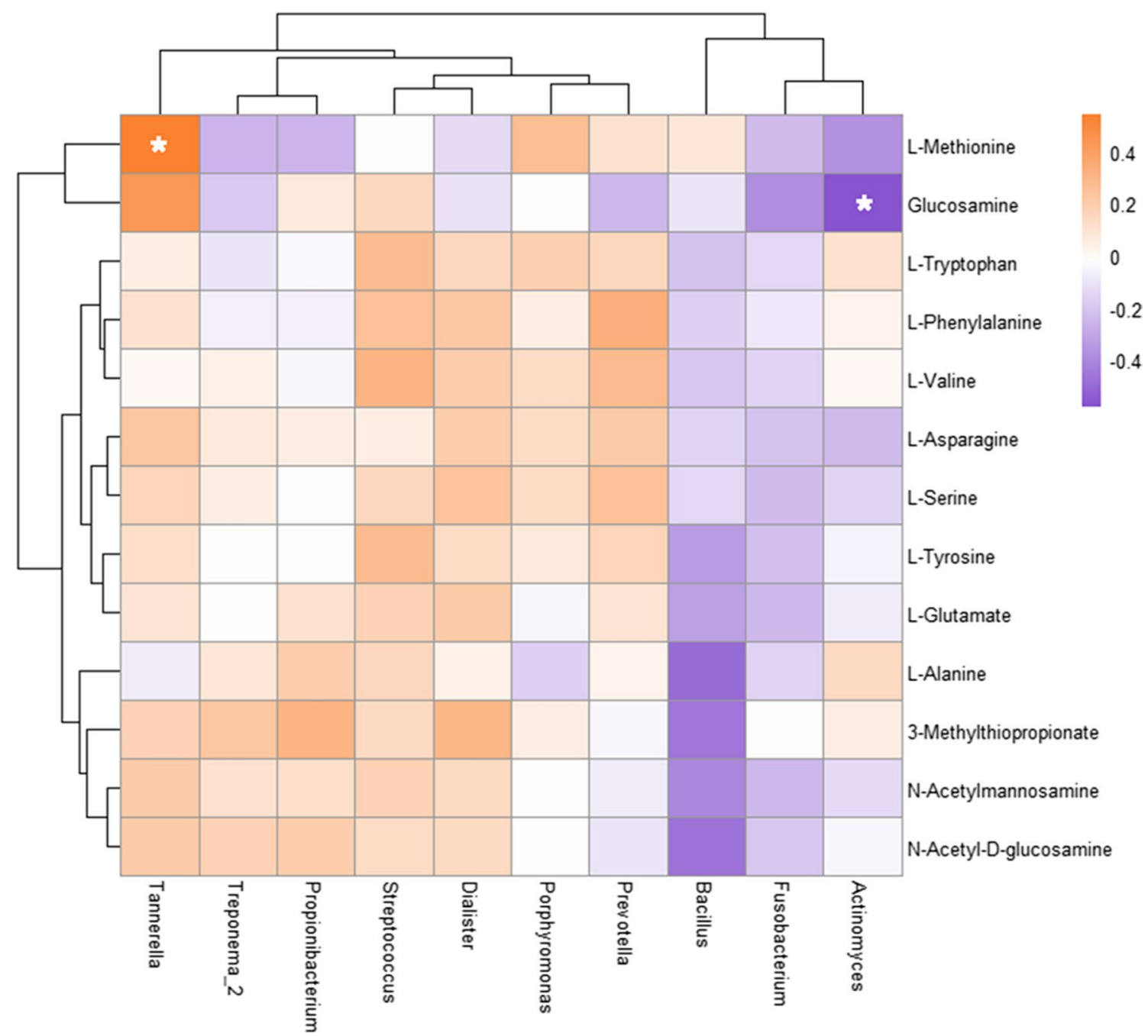

Fig. 11 Heatmap of correlation between major metabolites and relative abundance of major microorganisms in periapical periodontitis. Metabolite and microbial data were clustered using Spearman's correlation analysis. The correlation (R) value is expressed in different colors in the figure. The legend on the right is the color range of different $R$ values. ${ }^{*} p<0.05$

\section{Conclusion}

The study revealed that inflammatory grades are related to local metabolic changes in periapical periodontitis. The local changes in the metabolites GlcN and L-methionine were correlated with the changes in the abundance of the major microorganisms Actinomycetes and Tannerella, respectively. The findings provide important insights into local metabolome regulation during the progression of periapical periodontitis. However, further investigation is still required to explore the molecular interaction mechanisms underlying this issue.

\section{Abbreviations}

PCA: Principal components analysis; OPLS-DA: Orthogonal partial least squares discriminant analysis; LC-MS: UHPLC/QTOF-MS; QC: Quality control; VIP:
Variable importance in project; GlcN: Glucosamine; ManNAc: N-Acetylmannosamine; GlcNAc: N-acetyl-D-glucosamine.

\section{Acknowledgements}

Not applicable.

\section{Authors' contributions}

XW conceived the study, participated in study design, and was responsible for writing and submitting the final manuscript. YH and PZ participated in statistical analysis and drafted the manuscript. SQL and WD carried out the experimental studies related to metabolome and microbiome of chronic periapical periodontitis. QQZ and YL participated in sample collection in periapical periodontitis. All authors read and approved the manuscript.

\section{Funding}

This work was supported by the Foundation of the Priority Academic Program Development of Jiangsu Higher Education Institutions [Grant Number PAPD 2018-87]. The recipient of these funds is Xin Wei. 


\section{Availability of data and materials}

The datasets used and analysed during the current study are available from the corresponding author on reasonable request.

\section{Declarations}

\section{Ethics approval and consent to participate}

All clinical procedures performed in this study was approved by the Medical Ethics Committee of Jiangsu Stomatological Hospital (PJ-2018-032-001).

\section{Consent for publication}

Not applicable.

\section{Competing interests}

The authors declare that they have no competing interests.

\section{Author details}

${ }^{1}$ Jiangsu Province Key Laboratory of Oral Diseases, Department of Conservative Dentistry and Endodontics, Stomatological Hospital, Nanjing Medical University, Nanjing, China. ${ }^{2}$ Department of Operative Dentistry and Endodontics, Affiliated Stomatological Hospital of Nanjing Medical University, Nanjing, Jiangsu, China. ${ }^{3}$ Jiangsu Province Engineering Research Center of Stomatological Translational Medicine, Nanjing, China.

Received: 26 October 2021 Accepted: 5 November 2021

Published online: 23 November 2021

\section{References}

1. Gomes BP, Pinheiro ET, Jacinto RC, Zaia AA, Ferraz CC, Souza-Filho FJ: Microbial analysis of canals of root-filled teeth with periapical lesions using polymerase chain reaction. J Endod 2008, 34(5):537-540.

2. von Arx T. Apical surgery: a review of current techniques and outcome. Saudi Dent J. 2011;23(1):9-15.

3. Flynn TR, Shanti RM, Levi MH, Adamo AK, Kraut RA, Trieger N: Severe odontogenic infections, part 1: prospective report. J Oral Maxillofac Surg 2006, 64(7):1093-1103.

4. Gomes B, Herrera DR: Etiologic role of root canal infection in apical periodontitis and its relationship with clinical symptomatology. Braz Oral Res 2018, 32(suppl 1):e69.

5. Wang C, Zhang M, Guo H, Yan J, Liu F, Chen J, Li Y, Ren F. Human milk oligosaccharides protect against necrotizing enterocolitis by inhibiting intestinal damage via increasing the proliferation of crypt cells. Mol Nutr Food Res. 2019;63(18):e1900262.

6. Schefold JC, Zeden JP, Fotopoulou C, von Haehling S, Pschowski R, Hasper D, Volk HD, Schuett C, Reinke P: Increased indoleamine 2,3-dioxygenase (IDO) activity and elevated serum levels of tryptophan catabolites in patients with chronic kidney disease: a possible link between chronic inflammation and uraemic symptoms. Nephrol Dial Transplant 2009, 24(6):1901-1908.

7. Gupta R, Palchaudhuri S, Chattopadhyay D: Glutamate induces neutrophil cell migration by activating class I metabotropic glutamate receptors. Amino Acids 2013, 44(2):757-767.

8. Kouznetsova VL, Li J, Romm E, Tsigelny IF: Finding distinctions between oral cancer and periodontitis using saliva metabolites and machine learning. Oral Dis 2021, 27(3):484-493.

9. Tietz-Bogert PS, Kim M, Cheung A, Tabibian JH, Heimbach JK, Rosen CB, Nandakumar M, Lazaridis KN, LaRusso NF, Sung J, et al. Metabolomic profiling of portal blood and bile reveals metabolic signatures of primary sclerosing cholangitis. Int J Mol Sci. 2018;19(10):3188.

10. Tsai CH, Weng SF, Yang LC, Huang FM, Chen YJ, Chang YC: Immunohistochemical localization of tissue-type plasminogen activator and type I plasminogen activator inhibitor in radicular cysts. J Oral Pathol Med 2004, 33(3):156-161.

11. Li X, Zhao L, Wei M, Lv J, Sun Y, Shen X, Zhao D, Xue F, Zhang T, Wang J: Serum metabolomics analysis for the progression of esophageal squamous cell carcinoma. J Cancer 2021, 12(11):3190-3197.
12. Gupta A, Gupta S, Mahdi AA: (1)H NMR-derived serum metabolomics of leukoplakia and squamous cell carcinoma. Clin Chim Acta 2015, 441:47-55.

13. Zhang SL, Wang YP, Du ZX, Xue M, Zhang J, Ma Q. Lipid metabolomics in serum of hyperuricemic rats induced by fructose based on UPC(2)-Q/ TOF-MS. Zhongguo Zhong Yao Za Zhi. 2016;41(6):1135-9.

14. Wu YL, Lin AH, Chen $\mathrm{CH}$, Huang WC, Wang HY, Liu MH, Lee TS, Ru Kou Y: Glucosamine attenuates cigarette smoke-induced lung inflammation by inhibiting ROS-sensitive inflammatory signaling. Free Radic Biol Med 2014 69:208-218.

15. Vijayan V, Khandelwal M, Manglani K, Gupta S, Surolia A: Methionine down-regulates TLR4/MyD88/NF-kappaB signalling in osteoclast precursors to reduce bone loss during osteoporosis. Br J Pharmacol 2014, 171(1):107-121.

16. Yamada H, Akahoshi N, Kamata S, Hagiya Y, Hishiki T, Nagahata Y, Matsuura T, Takano N, Mori M, Ishizaki Y et al: Methionine excess in diet induces acute lethal hepatitis in mice lacking cystathionine gamma-lyase, an animal model of cystathioninuria. Free Radic Biol Med 2012, 52(9):1716-1726.

17. Zhang J, Bi J, Liu S, Pang Q, Zhang R, Wang S, Liu C. 5-HT drives mortality in sepsis induced by Cecal ligation and puncture in mice. Mediators Inflamm. 2017;2017:6374283.

18. Ozyel B, Le Gall G, Needs PW, Kroon PA. Anti-inflammatory effects of quercetin on high-glucose and pro-inflammatory cytokine challenged vascular endothelial cell metabolism. Mol Nutr Food Res. 2021:65(6):e2000777.

19. Islam J, Koseki T, Watanabe K, Budijanto S, Oikawa A, Alauddin M, Goto T, Aso $\mathrm{H}$, Komai $\mathrm{M}$, et al. Dietary supplementation of fermented rice bran effectively alleviates dextran sodium sulfate-induced colitis in mice. Nutrients. 2017:9(7):747.

20. Duan Y, Zhao Y, Zhu Q, Cai Q, Li H, Yin Y, Wang Z, Kong X: Dietary nutrient levels alter the metabolism of arginine family amino acids in the conceptus of Huanjiang mini-pigs. J Sci Food Agric 2019, 99(5):2132-2139.

21. Ma EH, Bantug G, Griss T, Condotta S, Johnson RM, Samborska B, Mainolfi $\mathrm{N}$, Suri V, Guak H, Balmer ML, et al. Serine is an essential metabolite for effector T cell expansion. Cell Metab. 2017;25(2):345-57.

22. Ma EH, Bantug G, Griss T, Condotta S, Johnson RM, Samborska B, Mainolfi $\mathrm{N}$, Suri V, Guak H, Balmer ML, et al. Serine is an essential metabolite for effector T cell expansion. Cell Metab. 2017;25(2):482.

23. Zhang H, Kovacs-Nolan J, Kodera T, Eto Y, Mine Y: gamma-Glutamyl cysteine and gamma-glutamyl valine inhibit TNF-alpha signaling in intestinal epithelial cells and reduce inflammation in a mouse model of colitis via allosteric activation of the calcium-sensing receptor. Biochim Biophys Acta 2015, 1852(5):792-804.

24. Liu Y, Bian Y, Luo X, Wang C, Mu D, Pan G, Wu J, Shi H: Synergistic effect of docosahexaenoic acid or conjugated linoleic acid with caffeic acid on ameliorating oxidative stress of HepG2 cells. J Food Sci 2021, 86(7):3240-3251.

25. Ferrucci L, Cherubini A, Bandinelli S, Bartali B, Corsi A, Lauretani F, Martin A, Andres-Lacueva C, Senin U, Guralnik JM: Relationship of plasma polyunsaturated fatty acids to circulating inflammatory markers. J Clin Endocrinol Metab 2006, 91(2):439-446.

26. Feng YL, Cao G, Chen DQ, Vaziri ND, Chen L, Zhang J, Wang M, Guo Y, Zhao YY: Microbiome-metabolomics reveals gut microbiota associated with glycine-conjugated metabolites and polyamine metabolism in chronic kidney disease. Cell Mol Life Sci 2019, 76(24):4961-4978.

27. Sridharan G, Ramani P, Patankar S: Serum metabolomics in oral leukoplakia and oral squamous cell carcinoma. J Cancer Res Ther 2017 13(3):556-561.

28. Barnes VM, Teles R, Trivedi HM, Devizio W, Xu T, Mitchell MW, Milburn MV, Guo L: Acceleration of purine degradation by periodontal diseases. J Dent Res 2009, 88(9):851-855.

29. Mussano F, Ferrocino I, Gavrilova N, Genova T, Dell'Acqua A, Cocolin L, Carossa S: Apical periodontitis: preliminary assessment of microbiota by $16 \mathrm{~S}$ rRNA high throughput amplicon target sequencing. BMC Oral Health 2018, 18(1):55.

30. Anderson AC, Al-Ahmad A, Elamin F, Jonas D, Mirghani Y, Schilhabel M, Karygianni L, Hellwig E, Rehman A. Comparison of the bacterial composition and structure in symptomatic and asymptomatic endodontic infections associated with root-filled teeth using pyrosequencing. PLoS ONE. 2013;8(12):e84960. 
31. Siqueira JF Jr., Rocas IN, Souto R, de Uzeda M, Colombo AP. Actinomyces species, streptococci, and Enterococcus faecalis in primary root canal infections. J Endod. 2002;28(3):168-72.

32. Ran S, Liu B, Gu S, Sun Z, Liang J: Analysis of the expression of NLRP3 and AIM2 in periapical lesions with apical periodontitis and microbial analysis outside the apical segment of teeth. Arch Oral Biol 2017, 78:39-47.

\section{Publisher's Note}

Springer Nature remains neutral with regard to jurisdictional claims in published maps and institutional affiliations.
Ready to submit your research? Choose BMC and benefit from:

- fast, convenient online submission

- thorough peer review by experienced researchers in your field

- rapid publication on acceptance

- support for research data, including large and complex data types

- gold Open Access which fosters wider collaboration and increased citations

- maximum visibility for your research: over 100M website views per year

At BMC, research is always in progress.

Learn more biomedcentral.com/submissions 